\title{
Case Report: Anaesthetic Management of Placenta Percreta
}

\author{
Subir Kumar Ghosh ${ }^{1}$, Babita Ramdev ${ }^{2}$, Noorjit Sidhu ${ }^{3}$, Archit Sharma ${ }^{4}$, \\ Arun Yadav $^{5}$, Madhav Choudhary ${ }^{6}$, Lalit Kumar ${ }^{7}$ \\ 1,3,5,6,7 Resident, ${ }^{2}$ Professor, Dept. of Anaesthesiology, MMIMSR, Mullana, Ambala-133203, India \\ ${ }^{4} \mathrm{GMCH}$, Sector-32, Chandigarh-160047, India \\ Corresponding Author: Babita Ramdev
}

DOI: https://doi.org/10.52403/ijrr.20220106

\begin{abstract}
Background: The placenta is a complicated organ and is partially understood. It is the essential part for physiological changes leading to a successful pregnancy. Placenta percreta is the most severe and least common form of placenta accreta in which villi penetrate the entire myometrial thickness and reach or traverse the serosa to encroach adjacent organs. Patients with placenta percreta are at a greater risk of life-threatening perioperative bleeding as well as massive and deadly thromboembolic events.
\end{abstract}

Case report: Our patient was a 34-year-old gravida 5female who underwent elective cesarean section at 37 weeks of gestation with a diagnosis of placenta accreta or percreta. Intraoperative findings showed placenta percreta with bladder wall involvement. Hence, hysterectomy was done. Anticipated intraoperative haemorrhage and hemodynamic instability were managed properly.

Discussion: Placenta percreta is the most serious among abnormal placentation, sometimes leading to catastrophic blood loss and very high maternal mortality and morbidity up to $10 \%$. The most important risk factor in placenta percreta is placenta previa (low lying placenta) after cesarean delivery. Our patient met all these risk factors. Prenatal diagnosis of an invasive placenta is paramount for reducing maternal morbidity and mortality by implementing a multidisciplinary approach.

Keywords: haemorrhage, placenta percreta, hysterectomy, high-risk pregnancy

\section{INTRODUCTION}

The placenta is a fascinating and complicated organ that is only partially understood because of its relatively short life span, fast growth, differentiation, and maturation. Its functions include the transportation of respiratory gases and metabolic wastes, immunologic protection, and the synthesis of steroid and protein hormones, among others. It is the only point of contact between the mother and her fetus, and it is essential in orchestrating maternal physiological changes that assure a successful pregnancy. ${ }^{[1]}$

Placenta accreta, placenta increta, and placenta percreta are three different types of abnormal placentation. ${ }^{[2]}$ Placenta percreta is the most severe and least common form of placenta accreta in which villi penetrate the entire myometrial thickness and reach or traverse the serosa to encroach adjacent organs with an incidence of 1.7 per 10,000 maternities overall. ${ }^{[3]}$

A past cesarean delivery in combination with placenta previa is the most significant risk factor for placenta accreta and the risk rises as the number of previous cesarean deliveries increases. Advanced maternal age, high parity, in-vitro fertilization, history of placenta accrete spectrum in a previous pregnancy, medical termination of pregnancy, manual removal of the placenta, uterine-artery embolization, and myomectomy are all identified as additional risk factors. ${ }^{[4]}$ 
Patients with placenta percreta are at a greater risk of life-threatening perioperative bleeding as well as massive and deadly thromboembolic events. ${ }^{[5]}$ All of these can increase postpartum morbidity and mortality with poor outcomes. ${ }^{[2]}$

Therefore, a multidisciplinary team approach is essential to manage such cases. The patient care team including nursing and blood bank staff, anesthesiologists, obstetricians, surgeons, urologists, neonatologists, and interventional radiologists becomes important to deal with the known complications and to improve the outcome of the mother and the baby. ${ }^{[6]}$

This is a case report of placenta percreta which was successfully managed with elective peripartum hysterectomy.

\section{CASE REPORT}

Our patient, a 34-year-old female with a history of amenorrhoea for 9 months came for a regular antenatal checkup and was admitted for safe confinement. She was $5^{\text {th }}$ gravida. Her first pregnancy was in 2009, during which she underwent elective lower segment cesarean section (LSCS) because of severe pre-eclampsia and post-dated pregnancy. She delivered a healthy female baby. In her second pregnancy, in 2012 she had full-term intrauterine fetal death which was delivered vaginally (VBAC). A year later (2013), she had 8 months of amenorrhoea and underwent emergency LSCS due to an abruptio placenta delivering a female child. She received two units of blood transfusion postoperatively. One and a half years ago in 2018, she had a spontaneous abortion at 4 months of gestation followed by dilatation and curettage.

In this pregnancy, she had conceived for the fifth time with a history of the midtrimester abnormal rise of blood sugar levels for which no treatment was taken. Abdominal ultrasonography done at $31^{+3}$ weeks showed polyhydramnios with low lying placenta reaching os. On admission, she had a blood pressure of 170/100 mm Hg and random blood sugar of 203mg/dl. An Internal Medicine opinion was taken for the management of gestational diabetes mellitus and hypertension. She was started on regular insulin injection as per the sliding scale. Intravenous labetalol 20mg was given immediately followed by oral labetalol 200mg thrice a day was prescribed. On day 3 of hospitalization, an ultrasonographic evaluation was done at 37weeks of gestation with an impression of cephalic presentation with an expected fetal weight of 3144grams with lower segment placenta with focal myometrial invasion suggestive of placenta accrete or percreta. Obstetrics ultrasonography also suggested uteroplacental and placentofetal insufficiency with fetal brain sparing effect. She was posted for elective cesarean delivery the next day.

A pre-anaesthetic evaluation for elective caesarean section and hysterectomy the next day was done. The patient was planned for surgery under general anaesthesia. Blood and blood products were arranged anticipating the risk of haemorrhage and the need for blood transfusion. In the preoperative ward, the patient's vitals were all within normal limits. Only one wide-bore peripheral venous access could be established on the left hand.

In the operating room, she was connected to multipara monitors with ECG, $\mathrm{SpO}_{2}$ and NIBP. The patient was induced with intravenous propofol and succinylcholine followed by laryngoscopy with C-Mac and intubation with a $7.0 \mathrm{~mm}$ internal diameter endotracheal tube. Maintenance of anaesthesia was done with oxygen, nitrous oxide, isoflurane, atracurium as required, and intermittent positive pressure ventilation. After induction arterial cannula was placed in the left radial artery for invasive blood pressure monitoring and an ultrasound-guided central line was inserted in the right internal jugular vein. Forced warm air was given to the patient for the prevention of hypothermia.

A midline skin incision was given above the umbilicus and the abdomen was opened in layers. Visual inspection of the 
uterus, bilateral adnexa, lower segment, extent of the bladder was done. An incision was given over the fundus of the uterus and the placenta was then found to be adherent to the anterior wall of the uterus, occupying the whole lower segment and upper segment. Breech extraction of the baby was done through the placenta. The baby was handed over to the paediatrician. The bladder wall was adherent to the lower uterine segment and intraoperative findings confirmed the bladder wall invasion by the placenta. Hence, cystostomy was done followed by bladder wall repair by the surgery team. Peripartum hysterectomy was performed.

During surgery, patient vitals were monitored carefully. Urine output and random blood sugar charting were done hourly. An estimated $2000 \mathrm{ml}$ of blood was lost during surgery. The patient received 3 units of packed red blood cells (PRBC) and 3 units of fresh frozen plasma (FFP) intraoperatively. Her vitals remained stable throughout the operative period. She was also given $10 \mathrm{ml}$ of intravenous calcium gluconate $10 \%$ slowly over 10 minutes. Total urine output mixed with blood during surgery was $550 \mathrm{ml}$. At the end of the procedure, the patient's vitals were stable with a clear chest on auscultation. The patient was safely extubated and shifted to the postoperative ward for monitoring. Postoperatively the patient was hemodynamically stable. Blood samples were sent for complete blood count, serum electrolytes, and arterial blood gas analysis. Hourly blood sugar monitoring was continued and was managed accordingly. The postoperative period was uneventful.

\section{DISCUSSION}

Abnormally invasive placentas are a life-threatening condition characterized by placental villi being abnormally adherent to the myometrium as a result of the absence of, or defects in, the normal decidual basalis and the fibrinous Nitabuch's layer. ${ }^{[7]}$ This includes placenta accrete in which placental chorionic villi invade only superficial myometrium, placenta increta where placental chorionic villi invade more than half of the myometrium, and very rarely, placenta percreta where chorionic villi invade through the myometrium, serosa, and occasionally into adjacent organs such as the bladder. ${ }^{[8]}$ Placenta percreta is the most serious among abnormal placentation, sometimes leading to catastrophic blood loss and very high maternal mortality and morbidity up to $10 \% .{ }^{[9]}$

The risk of Placenta Accrete Spectrum disorders in women with a placenta previa and previous cesarean deliveries are $3 \%, 11 \%, 40 \%, 61 \%$, and $67 \%$ for the first, second, third, fourth, and fifth and more cesareans respectively. Over the last 40 years, cesarean section rates have risen globally from less than $10 \%$ to over $30 \%$, and at the same time a 10 -fold increase in the incidence of accrete syndromes. ${ }^{[4]}$

Our patient had a low-lying placenta and also had undergone two previous cesarean sections and one dilatation and evacuation. The most important risk factor in placenta percreta is placenta previa (low lying placenta) after cesarean delivery. Other risk factors for placenta percreta include submucous myoma, previous curettage, Asherman's syndrome, advanced maternal age, grand multiparity, smoking, chronic hypertension as well as a previous history with adherent placenta disorders. ${ }^{[10]}$

Prenatal diagnosis of an invasive placenta is paramount to reduce maternal morbidity and mortality as it allows planning and timing of the surgery, arrangements for interventional radiology, availability of blood products and cell salvage, plan for the most appropriate surgical technique, and support from other specialities if there is any involvement of the ureters, bladder or bowel. ${ }^{[11]}$ Ultrasound findings suggestive of placenta accreta spectrum disorders include the appearance of multiple intra-placental lacunae, turbulent flow of blood in the lacunae, loss or absence of the retro-placental clear space between the uterus and placenta, disruption of the 
posterior bladder wall-myometrial interface, reduction in myometrial thickness to $<1 \mathrm{~mm}$, hyper-vascularity and irregularity of the adjacent bladder wall. ${ }^{[12]}$

For diagnosis of a posterior placenta, placenta percreta, and for imaging morbidly obese patients MRI is essential. Loss of myometrium overlying the placenta, placental tissue extending to the bladder, heterogeneous signal intensity in the placenta, focal areas of uterine bulging, and loss of interface with adjacent organs are features of placenta percreta on nonenhanced MRI. ${ }^{[13]}$ Our patient had undergone ultrasonography which revealed anterior low-lying placenta, with features of placenta accreta or percreta with uteroplacental and placentofetal insufficiency with fetal brain sparing effect.

Both abnormal adherence and invasion of villous tissue into the myometrium result in failure of spontaneous separation of the placenta from the uterine wall at delivery. When unsuspected at the time of delivery, attempts to manually remove accreta villous tissue typically provoke rapid bleeding from the uteroplacental circulation. In invasive cases, this can lead to massive obstetric haemorrhage due to the disruption of the deep uterine vasculature of the increta or percreta area. ${ }^{[14]}$ Reports indicate that there is a greater benefit and decrease in mortality for planned hysterectomy versus an emergent peripartum hysterectomy in these patients. Also, a significant reduction in morbidity and mortality is reported with the utilization of a multidisciplinary team, comprising of an obstetrician experienced in pelvic surgery, a blood bank team, an anesthesiologist, a urologist skilled in pelvic reconstruction, an interventional radiologist, and an experienced neonatologist towards the successful treatment of placenta previa. ${ }^{[10]}$

In pregnancies with placental anomalies, the standard delivery date is generally the $34^{\text {th }}$ gestational week. However, several studies recommend delivery at 34-36 weeks in stable patients and 32-34weeks as appropriate in pregnancy with a history of bleeding and delivery before 32 weeks only in emergency cases. ${ }^{[15]}$ Our patient underwent surgery at a period of gestation of $36^{+4}$ weeks with no steroid coverage as it has already crossed the age for lung maturity. The delay in the surgical procedure was due to the preoperative optimization of the patient's medical condition.

Our patient was electively given general anaesthesia for the procedure. Patients with placental invasion defects require special attention when it comes to anaesthetic management. The best anaesthetic technique should be chosen with care. ${ }^{[16]}$ In such cases, general anaesthesia is the preferred mode. This preference can be explained by the increased risk of bleeding associated with the loss of clotting factors and rapidly deteriorating hemodynamics in a short time; additionally, hemodynamics can be worsened by a sympathetic blockage in regional anaesthesia. Furthermore, bleeding and increased surgical manipulation prolong the operating time, causing discomfort in the awake patient. ${ }^{[15]}$

$$
\text { Planned preterm (34-35weeks) }
$$

cesarean hysterectomy with placenta left in situ is the recommended management strategy for Placenta Accreta Spectrum (PAS) by the American College of Obstetricians and Gynecologists (ACOG). [17] To prevent maternal morbidity from blood loss from the placental bed, the uterine incision should be given avoiding placental transection. ${ }^{[4]}$ In our case also cesarean hysterectomy was done and the uterine incision was given over the fundus. However, breech extraction was done through the placenta as the placenta was found to be adherent to the anterior wall of the uterus occupying the whole lower and upper segment. Because of the possibility of malignancy developing in the cervical stump, the requirement for regular cervical cytology, and other complications like bleeding or discharge per vagina, total peripartum hysterectomy is the preferable approach. ${ }^{[17]}$ 
Massive haemorrhage during delivery and total hysterectomy makes it critical to know how to respond to massive haemorrhage and DIC in a timely and suitable manner. It is necessary to make arrangements for an adequate number of health care providers, role division, access to the venous system, blood transfusion, hemostatic technique, and data collection must all be coordinated in parallel. In critical massive haemorrhage cases, Takeda $S$ et al. recommended, the blood of the same ABO group be used, even omitting the cross-matching test. In case of insufficient availability of the same ABO group, noncrossmatched compatible RBC and fresh frozen plasma (FFP) can be used. Transfusions of RBC and FFP should be done to achieve haemoglobin levels of 7 to $8 \mathrm{~g} / \mathrm{dl}$, fibrinogen levels of $150 \mathrm{mg} / \mathrm{dl}$ or higher, and total protein levels of $4.0 \mathrm{~g} / \mathrm{dl}$ or higher. The platelet concentration should be kept at or above 100000/cubic millimetres. [18]

In our case patient had crossed $36 \mathrm{wks}$ and her haemoglobin was $13.4 \mathrm{~g} / \mathrm{dl}$ with pre-eclampsia which could be due to hemoconcentration. Intraoperatively 3 units of packed RBC (PRBC) and 3 units of FFP were transfused as estimated loss in peripartum hysterectomy was $2000 \mathrm{ml}$.

\section{CONCLUSION}

A 34 years old $5^{\text {th }}$ gravida with an antenatal diagnosis of placenta accreta spectrum underwent elective caesarean delivery and hysterectomy. Her previous history of multiple caesarean deliveries, placenta previa made her more prone to the development of placenta percreta. Preemptive planning anticipating massive intraoperative haemorrhage and hemodynamic instability was the key for managing such patients in the perioperative period. Our patient was managed effectively and successfully perioperatively.

\section{Financial support and sponsorship: Nil}

Acknowledgement: None

\section{Conflict of Interest: None}

\section{REFERENCE}

1. Gabbe SG, Niebyl JR, Galan HL, Jauniaux ERM, Landon MB, Simpson JL, et al. Obstetrics: Normal and problem pregnancies E-book. 6th ed. Saunders; 2016. $02 \mathrm{p}$.

2. Dutta S, Dey B, Chanu S, Marbaniang E, Sharma N, Khonglah Y, et al. A retrospective study of placenta accreta, percreta, and increta in peripartum hysterectomies in a tertiary care institute in Northeast India. Cureus. 2020;12 (11): e11399

3. Lee, P. S., Kempner, S., Miller, M., Dominguez, J., Grotegut, C., Ehrisman, J., Previs, R., Havrilesky, L. J., Broadwater, G., Ellestad, S. C., \& Secord, A. A. (2017). Multidisciplinary approach to manage antenatally suspected placenta percreta: updated algorithm and patient outcomes. Gynecologic Oncology Research and Practice, 4(1), 11.

4. Morlando M, Collins S. Placenta accreta spectrum disorders: Challenges, risks, and management strategies. Int $\mathrm{J}$ Womens Health. 2020;12:1033-45.

5. Atallah D, Abou Zeid H, Moubarak M, Moussa M, Nassif N, Jebara V. "You only live twice": multidisciplinary management of catastrophic case in placenta Accreta Spectrum-a case report. BMC Pregnancy Childbirth. 2020; 20(1):135.

6. Riveros-Perez E, Sanchez MG, Odo N, Shukla M, Rungruang B. Supra-massive transfusion and interdisciplinary approach to cesarean hysterectomy due to complex placenta percreta: Case report. SAGE Open Med Case Rep. 2021

7. Sentilhes L, Goffinet F, Kayem G. Management of placenta accreta. Acta Obstet Gynecol Scand. 2013;92(10):112534.

8. Tillu N, Savalia A, Patwardhan S, Patil B. Placenta percreta with bladder invasion: The armamentarium available in its management. Urol Ann. 2019;11(3):324-7.

9. Heena AB, Kumari G. Retrospective study of placenta accreta, placenta increta and placenta percreta in Peripartum hysterectomy specimens. Indian J Pathol Microbiol. 2020;63(Supplement):S87-90.

10. Ibrahim MA, Liu A, Dalpiaz A, Schwamb R, Warren K, Khan SA. Urological 
manifestations of placenta percreta. Curr Urol. 2015; 8(2):57-65.

11. Piñas Carrillo A, Chandraharan E. Placenta accreta spectrum: Risk factors, diagnosis and management with special reference to the Triple $\mathrm{P}$ procedure. Womens Health (Lond Engl). 2019; 15:1745506519878081.

12. Okunowo AA, Ohazurike EO, HabeebuAdeyemi FM. Undiagnosed placenta praevia percreta: A rare case report and review of management. Niger Postgrad Med J. 2019;26(1):61-4.

13. Sivasankar C. Perioperative management of undiagnosed placenta percreta: case report and management strategies. Int $\mathrm{J}$ Womens Health. 2012;4:451-4.

14. Jauniaux E, Grønbeck L, Bunce C, Langhoff-Roos J, Collins SL. Epidemiology of placenta previa accreta: a systematic review and meta-analysis. BMJ Open. 2019;9(11):e031193. PA

15. Urfalıglu A, Öksüz G, Bilal B, Teksen S, Calıșır F, Boran ÖF, et al. Retrospective evaluation of anesthetic management in cesarean sections of pregnant women with placental anomaly. Anesthesiol Res Pract. 2020; 2020:1358258.

16. Binici O, Büyükfirat E. Anesthesia for cesarean section in parturients with abnormal placentation: A retrospective study. Cureus. 2019; 11(6):e5033.

17. Jauniaux E, Hussein AM, Fox KA, Collins SL. New evidence-based diagnostic and management strategies for placenta accreta spectrum disorders. Best Pract Res Clin Obstet Gynaecol. 2019;61:75-88.

18. Takeda S, Takeda J, Makino S. Cesarean section for placenta previa and placenta previa accreta spectrum. Surg J. 2020; 6(Suppl 2):S110-21.

How to cite this article: Ghosh SK, Ramdev B, Sidhu $N$ et.al. Case report: anaesthetic management of placenta percreta. International Journal of Research and Review. 2022; 9(1): 39-44. DOI: https://doi.org/10.52403/ijrr. 20220106 